\title{
Amphitheater of Volterra: Case Study for the Representation of Excavation Data
}

\author{
CARLO BATTINI, University of Genoa, Italy \\ ELENA SORGE, Soprintendenza Archeologia della Toscana, Italy
}

\begin{abstract}
The present paper aims to describe how different surveying techniques can be used together for a better understanding of the artefact under investigation. Digital surveying employing tools such as terrestrial laser scanning (TLS) and Structure-from-Motion (SfM) software can be used together and made to interact with one another in order to compile an exhaustive database, rich in colorimetric and metric information.

The case study examined in the present research is the discovery of the Amphitheater of Volterra. It was discovered in July 2015 during the clearance operation of a local stream, and it is located near Porta Diana, a few hundred meters from the Roman Theater, which was discovered in the last century. The excavation campaign occurred between October and November 2015, and it revealed the ridges of the structure's support walls. This structure is constituted by three levels, which together are nearly ten meters deep.

In the post-processing phase, three-dimensional models were used to create the metric images necessary to study the stratigraphic units. Moreover, during this phase, it was possible to test the ability of mobile applications to visualize and manage 3D models and excavation information.
\end{abstract}

Key words:

Mobile App, Virtual, Amphitheater, 3D Models, Digital Survey.

SDH Reference:

Carlo Battini and Elena Sorgel. 2017. Amphitheater of Volterra: case study for the representation of

the excavation data, SDH, 1, 2, 269-281.

DOI : $10.14434 /$ sdh.v1i2.23242

\section{INTRODUCTION}

The present paper aims to describe an experimental method to organize the data gathered during an archaeological excavation. Using different surveying techniques simultaneously can contribute to a better understanding of the studied artefact on variable levels of detail. After having been validated, the gathered data were inserted into a database managed by mobile systems. The present paper studies a prototype of a mobile application, which is currently being implemented, to organize information with virtual visualizations. Features of the project include adding textual information, saving graphic notes, executing linear measurements, and taking photographs localized in the virtual three-dimensional space.

Author's address: Carlo Battini, DICCA - Department of Civil, Chemical and Environmental Engineering, University of Genoa, 16145, Via Montallegro 1, Genoa, Italy; email: carlo.battini@unige.it; Elena Sorge, Soprintendenza Archeologia della Toscana Florence, Italy.; email: elena.sorge@beniculturali.it

Permission to make digital or hardcopies of part or all of this work is granted without fee according to the open access policy of SDH.

(C) 2017 SDH Open Access Journal

Studies in Digital Heritage, Vol. 1, No. 2, Publication date: December 2017 


\section{THE DISCOVERY OF THE AMPHITHEATER}

The Amphitheatre of Volterra was rediscovered on the 8th July 2015, with the uncovering of a 40meter-long curved wall (Fig. 1, Fig. 2). Thanks to the funding from the Bank of Volterra, it was possible to start the first excavation campaign, which confirmed the hypothesis that this structure was actually a forgotten Roman amphitheater consisting of three floors (Fig. 3).

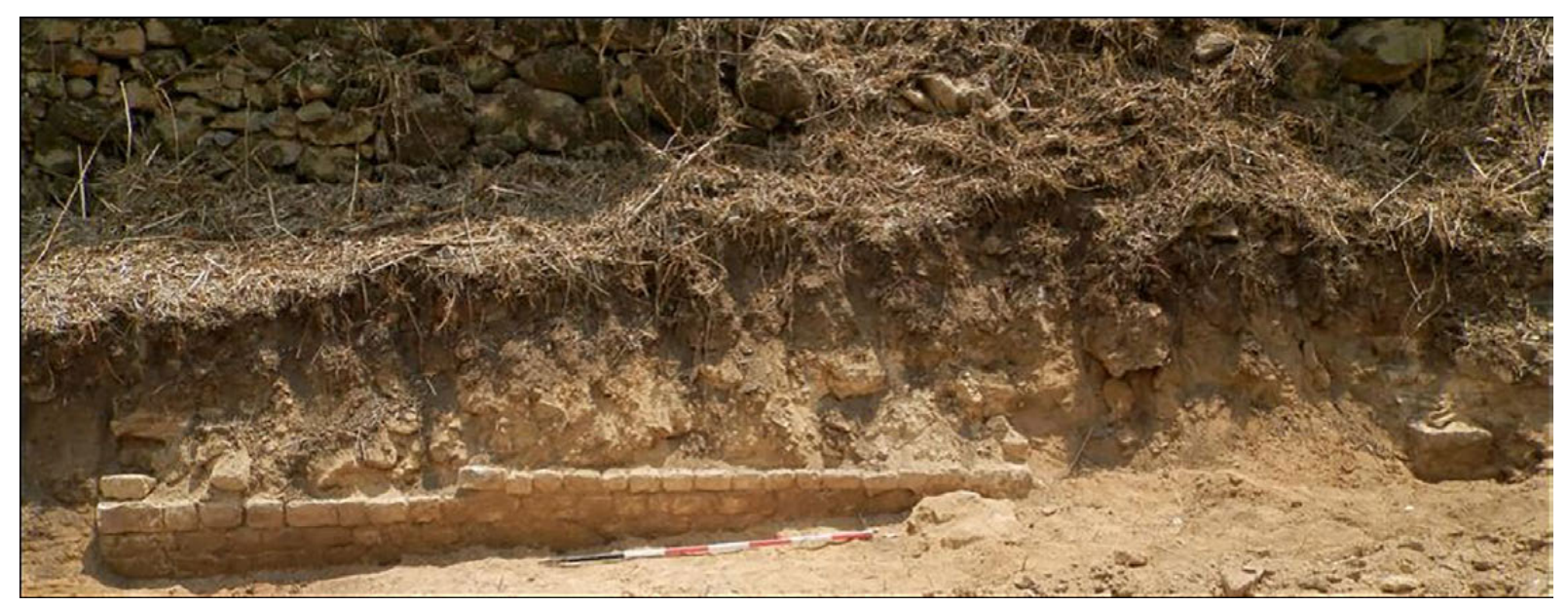

Figure 1. The discovery of the first stretch of Roman masonry.

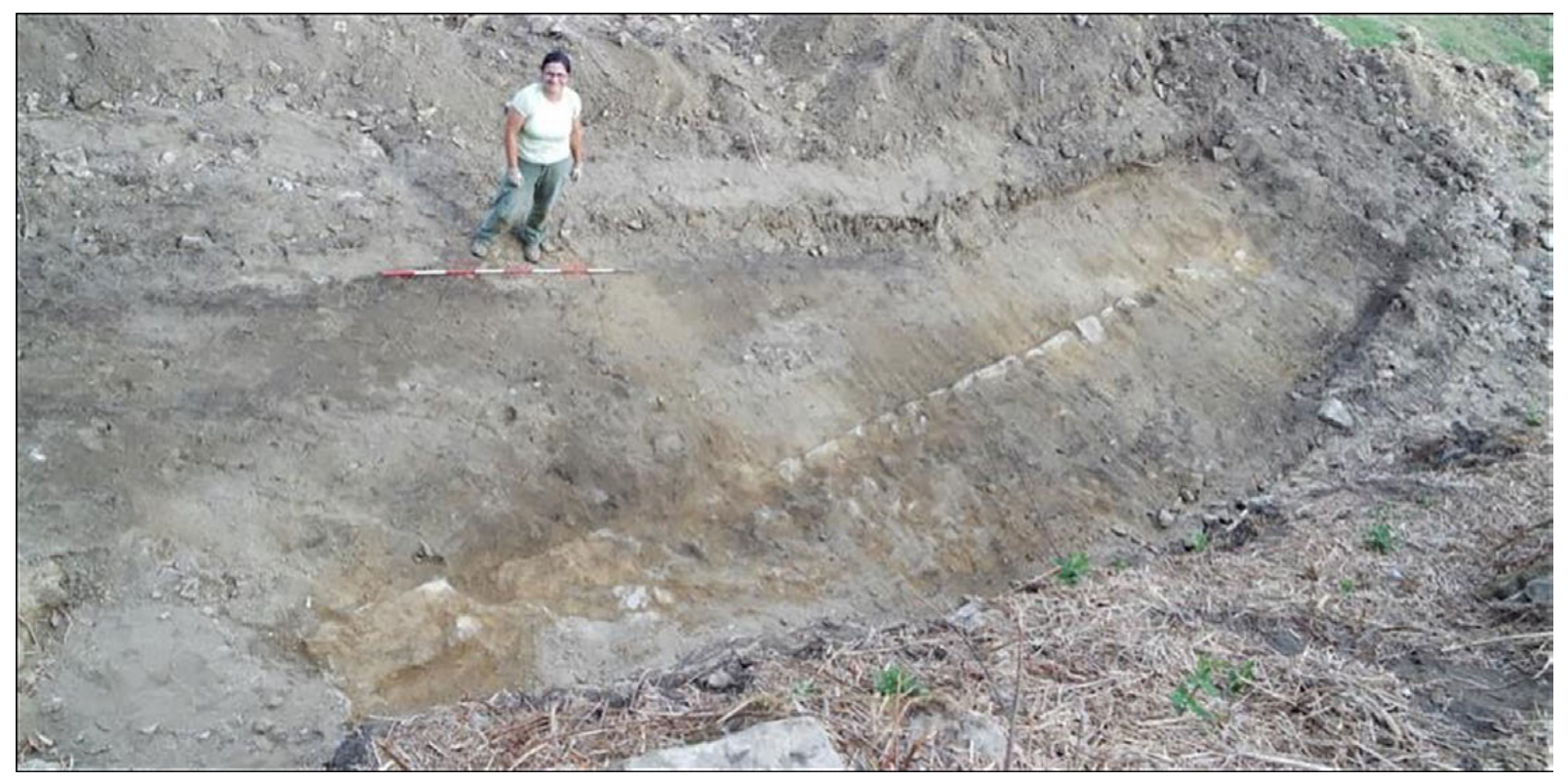

Figure 2. The curved line of the wall is visible. At this stage of the work, the presence of a Roman amphitheater was hypothesized. 


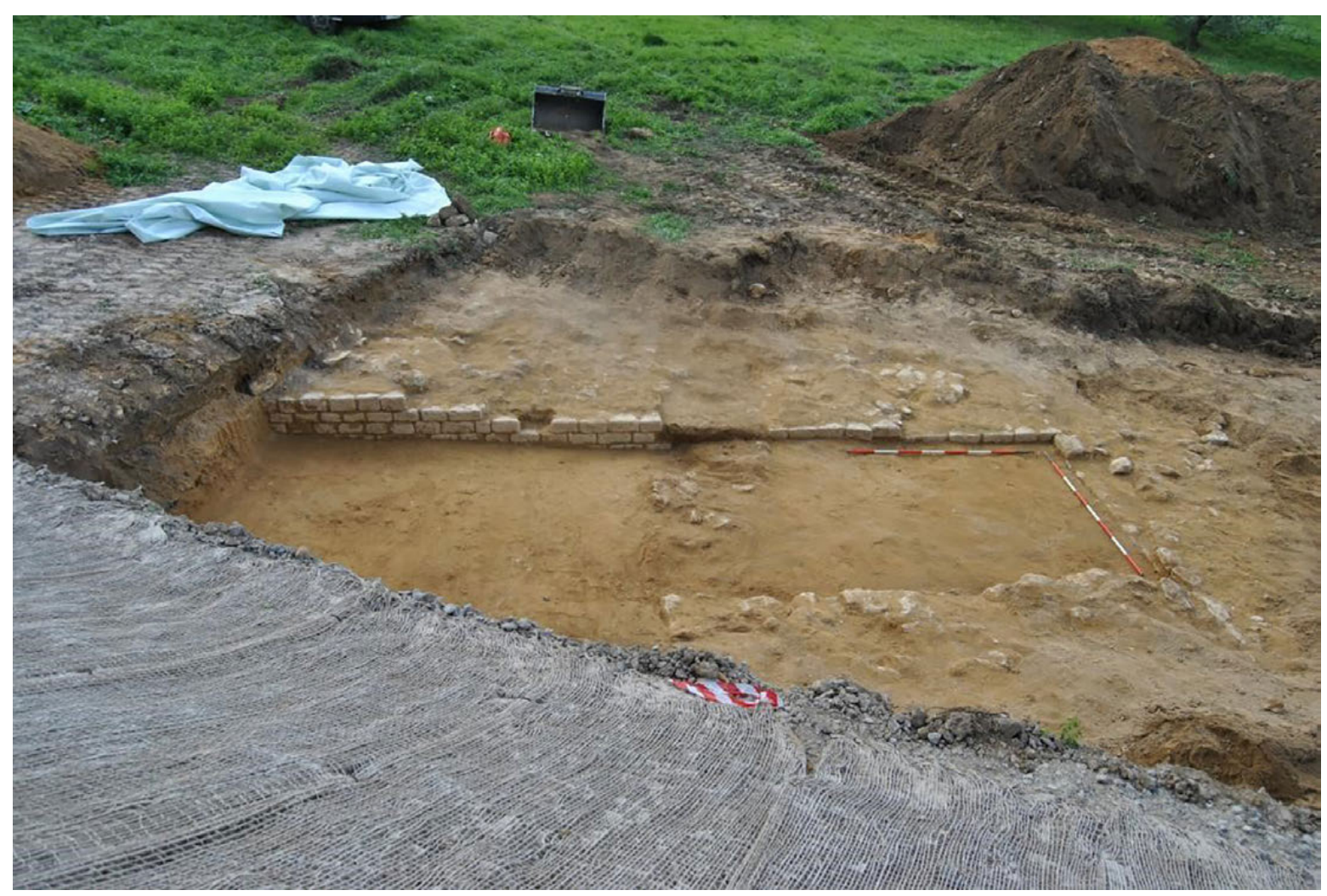

Figure 3. After just one day of excavation, archaeologists found the first confirmation of the presence of a structure hidden in the ground.

The aim of our first season, which lasted six weeks, was primarily to confirm the existence of the monument itself, as well as to plan the subsequent excavation campaigns, which were postponed as winter was drawing near. For this reason, only the first layers, were removed, thus revealing the actual dimensions of the amphitheater. This sample provided the concrete evidence that the structure discovered was, in fact, an amphitheater composed of three floors, built with steps, which, however, were not found in this section. The cavea was vast and structured on three levels, prima, secunda, and summa cavea (or Maenianum primum, secundum, and summum), separated by the praecinctiones, which can be described as thick concentric support walls. The arena is located at a height that we imagine to be two metres below where we reached at the end of the excavation; therefore, it should be circa 6 meters from the height of the current excavation level (Fig. 4). Architecturally, the amphitheater could likely have had a mixed structure, with strong similarities to the Vallebuona theatre, i.e., inserted (where possible) into the slope of a hill, with other parts built on substructures, as research carried out in one of its carceres (chariot pit) seemed to prove.

During the first survey, it was not possible to examine the ruins; therefore, currently nothing can be said about the actual state of conservation of the structure. The non-invasive geo-diagnostic surveys, carried out during winter in the area by SOING, a company based in Livorno, Italy and a project 
partner of the Italian Superintendency of Cultural Heritage, showed that the structure was even larger than previously suspected (Fig 5).



Figure 4. The portion of the cavea of the amphitheater with the carceres of the first two orders delimited by the first and second praecinctio.

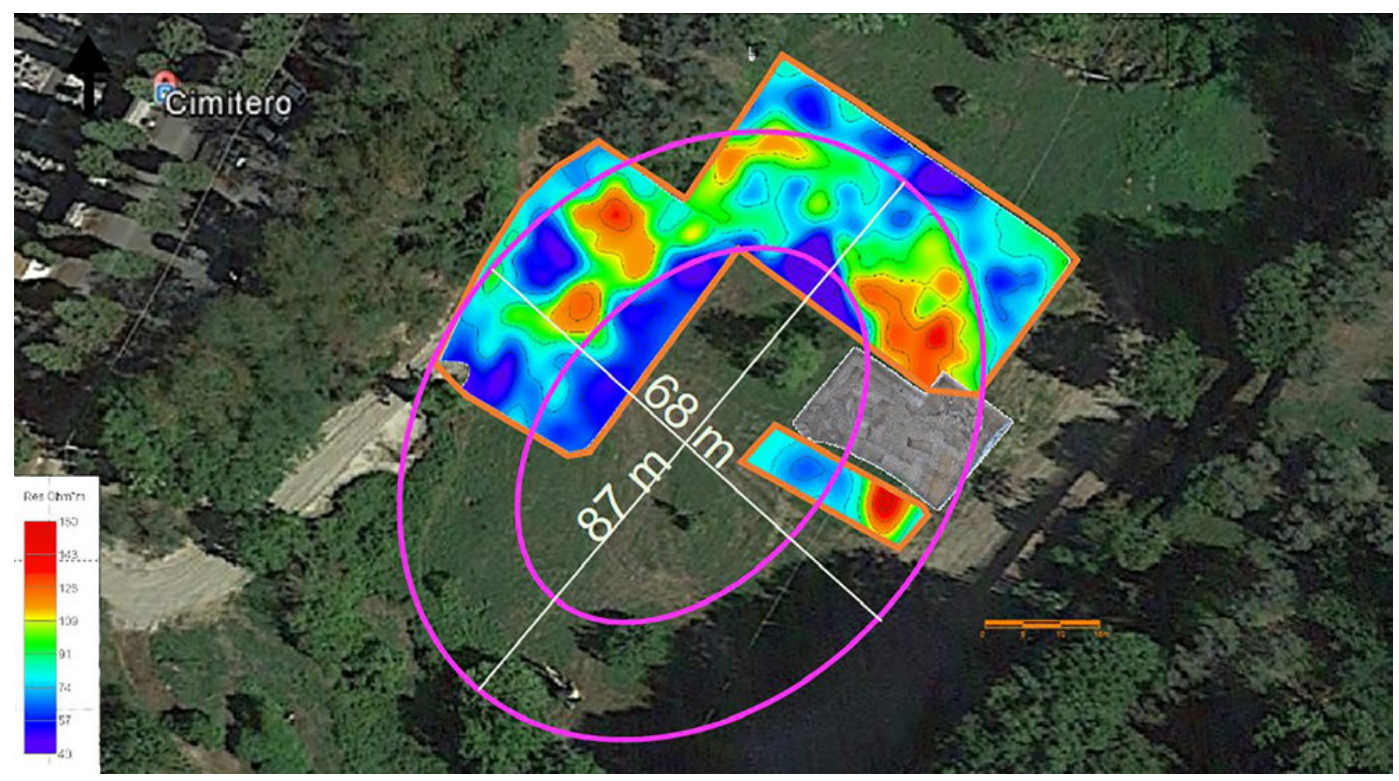

Figure 5. Image of the non-invasive geo-diagnostic surveys carried out during winter in the area by SOING (Livorno, Italy). 
Thanks to a collaboration with the Department of Civil, Chemical and Environmental Engineering (DICCA) in Genoa, it was possible to carry out digital surveys to study the samples taken. The reconstruction of the monument showed that its dimensions are around $82 \mathrm{~m} \times 64 \mathrm{~m}$, with three stone step floors, as demonstrated in the last excavated area (Fig. 6).

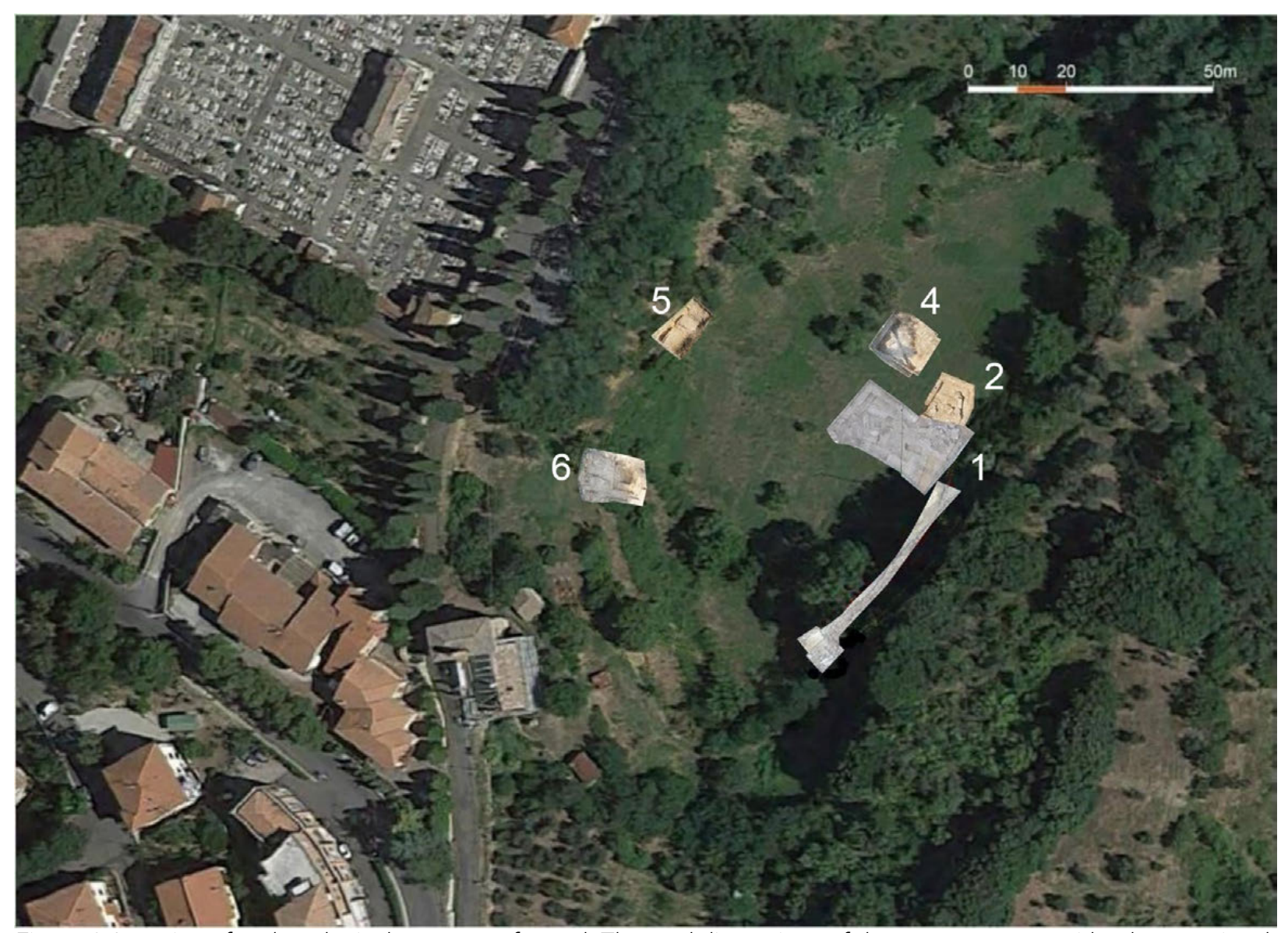

Figure 6. Location of archaeological assays performed. The total dimensions of the monument are wider than previously hypothesized - they seem to be around $82 \mathrm{~m} \times 64 \mathrm{~m}$.

\section{EXCAVATION AND RECORDING INFORMATION}

The excavation procedures were implemented using modern techniques of three-dimensional surveying, which can acquire a vast amount of data to then create a rich database for investigating the finds. Nowadays, these methods are essential for data interpretation, conservation, and storage, as well as the appreciation of the finds, thanks to interacting systems of visualization [Russo et al. 2011].

As usually happens, the techniques of digital surveying, i.e., terrestrial laser scanning (TLS), topography and the Structure-from-Motion (SfM) technique [Bertocci 2014] can be used together to 
acquire the most information possible, depending on the peculiarities of the utilized technique. These surveying techniques have different levels of definition and errors. However, they can be compared and used simultaneously to enrich the database with new information. In the survey of the Amphitheater of Volterra, the three techniques were implemented in accordance with a project of precise survey, which was fundamental for analyzing the quality of the data collected as well as for defining an operative procedure for the campaign of data acquisition. In the first phase, a topographic support network was built to record the data coming from the TLS (Fig. 7) and SfM surveying techniques [Russo et al. 2011].


Figure 7. The first laser scanner survey campaign: a) scanner $Z+F$ Imager 5006h; b) point cloud displayed within the Leica Cyclone 9.1 software.

Several scientific contributions have studied these two methods, which today are consolidated in the discipline of surveying, with the aim of determining the potential strengths and limitations of using them in alternation or at the same time [Beraldin 2004; Boehler and Marbs 2004]. The application of these methods in the field of Cultural Heritage [Boehler and Marbs 2004; Grussenmeyer et al. 2008] highlights how some parameters are fundamental to a successful survey. Dimension, geometric complexity, and coloration can be pivotal factors in increasing or diminishing measurement errors at the moment of the three-dimensional acquisition. Therefore, particular attention was given to the definition of a maximum admissible error. Owing to the presence of sandy soil and ashlar walls and because of the dimension of the structures to be surveyed, the maximum allowable error was set at $1.5 \mathrm{~cm}$. With this value, it was possible to store all the information on the collected stratigraphic units as well as to operate with an expeditious approach during the stage of survey. The processes leading to the evaluation of this error included the comparison of the databases acquired with the two different surveying techniques. For the comparative test, the decision was made to analyze the result of the first excavation campaign, which was characterized by extreme geometrical complexity as well as by the presence of areas with soil and well-preserved wall structures.

Using the Z+F Imager 5006h phase-shift laser, which can scan structures within a range of 79 metres with an accuracy of $2 \mathrm{~mm}$, it was possible to record the geometrical structures of the archaeological site with the reflectance value of the material. The point clouds, which were needed to create a three- 
dimensional model of the sample, were acquired using 14 laser locations. The point clouds were converted into a 3D-mesh model by simplifying data with a distance of $1 \mathrm{~cm}$ between all points (a value below the acceptable error defined in the preliminary stage). Subsequently, the resulting threedimensional model underwent a process of cleaning and elimination of all imperfections. The final result was a model of 12,058,625 triangles (Fig. 8).

At the same time, the 3D data of this excavation area were captured using the SfM technique. The surveying process was carried out with a Nikon D5000 reflex camera using an 18-105 mm lens. By maintaining the same focal aperture, it was possible to acquire a great number of shots, which were subsequently elaborated using Photoscan (version 1.2.2; Fig. 9).

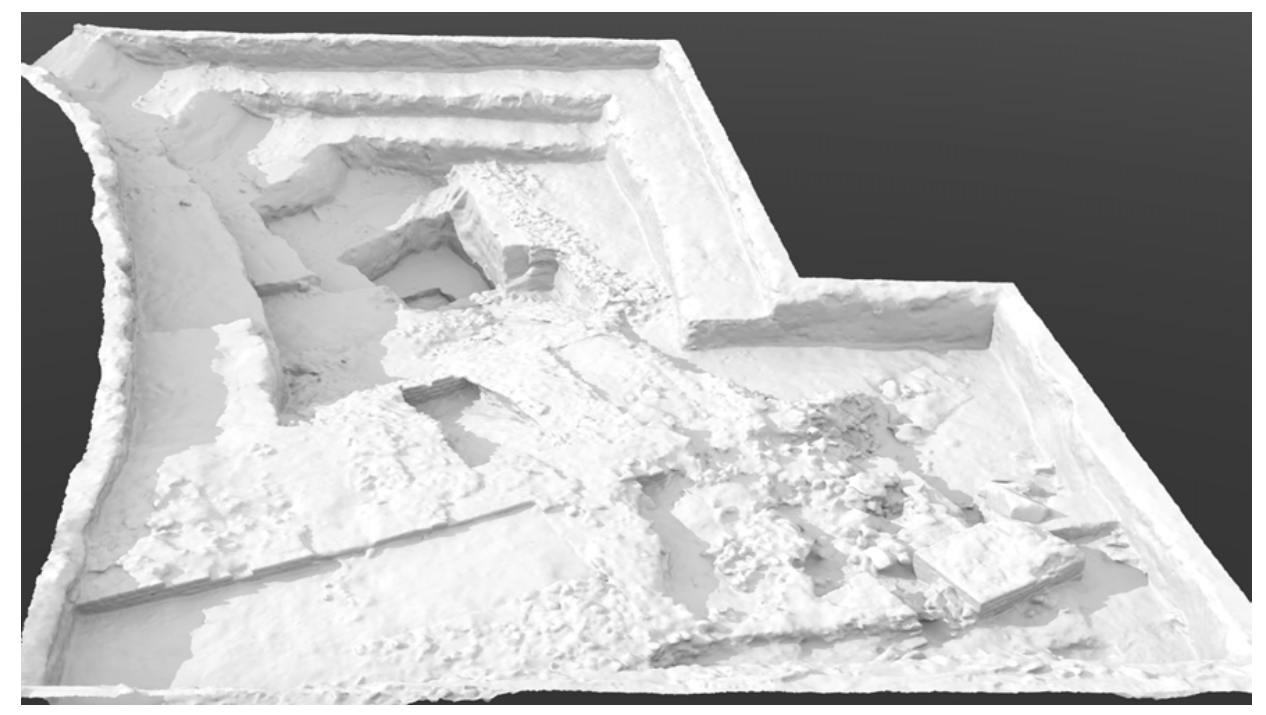

Figure 8. Three-dimensional model composed of approximately 6 million polygons deriving from the points cloud acquired with laser scanner.



Figure 9. The acquired images (all shot with the same focal length) were used to reconstruct the threedimensional model with the SFM technique. 
After the processes of image alignment, and the construction of a sparse cloud, a dense cloud, a 3D model and texture, it was possible to create a 3D model with the same orientation and dimensions as the result obtained with the TLS technique. The two three-dimensional models were compared using the CloudCompare 2.6.2 software, in order to evaluate the differences between the two surveying techniques. The values acquired were a mean-distance value of $0.002917 \mathrm{~m}$ and the standard-deviation value of $0.012793 \mathrm{~m}$, which are both below the limit defined during the preliminary phase (Fig. 10).

Based on this comparison, for the following three-dimensional surveys the research team decided to adopt the SfM technique (with topographic support) as the main tool to acquire metrical information. This technique proved to be less expensive in the phase of data elaboration and could also be used by less experienced staff, which avoided excessive interruption of the excavation processes.



Figure 10. The comparison of the two three-dimensional models (TLS and SfM) within the open-source software CloudCompare 2.6.2.

\section{MOBILE APPLICATION FOR DATA MANAGEMENT}

The collection of information is only the first step in the understanding an archaeological object. Data must be catalogued and made available to several experts of various disciplines for interpreting the historical evolution of the site. The archaeological excavation is the moment in which all information not collected and catalogued will be definitively lost, disrupting the process of understanding of the object investigated [Bezzi et al. 2011]. Collecting and recording the information at regular intervals is, therefore, the first step to scientifically validate the information collected [Grabner et al. 2003]. 
New research projects can be developed with the use of commercial platforms and open source tools, such as three-dimensional models, images, texts, and sounds to view and interact with the collected data. Many studies, such as the Archeoguide [Vlahakis et al. 2002], were able to create guided tours in augmented reality with head-mounted displays. Other projects, such as Virtual Rome, reconstructed the landscape of modern day Rome and that of Rome in the second century A.D. using common web browsers with three-dimensional online reproduction, including multimedia insights [Pescarin et al. 2009].

Currently, the 3D Heritage Online Presenter (3DHOP) platform, developed by the Visual Computing Laboratory of the Department Isti-CNR of Pisa, proves to be a promising project for visualizing digitized media assets on the web [Potenziani et al. 2015]. The use of multiresolution encoding efficiently streams high-resolution 3D models, such as the sampled models usually employed in Cultural Heritage applications. In addition, it provides a series of ready-to-use templates and examples tailored to the presentation of $\mathrm{CH}$ objects, and it interconnects the 3D visualization with the rest of the DOM webpage, making it possible to create integrated presentation schemes (3D and multimedia).

The present research aims to determine how a mobile application can aid the understanding and the study of an archaeological object. In particular, the use of virtual reality can be an essential tool to understand the spatial morphology of the artefact, allowing the user to use and add information pivotal to the understanding of the object's evolution. The aim is to create an application able to:

- $\quad$ Three-dimensionally browse within the 3D models of the collected samples;

- $\quad$ Take measurements from one point to the other;

- $\quad$ Record textual and graphical notes located in the 3D space, in order to facilitate the study of the object;

Take photos using the camera of the mobile system, and locate these images in specific points of the 3D model.

The present project was realized using Unity 3D, a programming software usually adopted to develop videogames and able to provide all the necessary utilities to create a 3D environment. The development of the proposed application started by defining the features necessary to support user interaction. The application should visualize three-dimensional models, enable virtual browsing, and organize text and images. Data can already be in the application, or it can be inserted during runtime by the user while browsing. In order to promote potential developments of this application and to give it greater versatility, the decision was made to create an SQL database which can be updated within the server to exchange and save the collected information (Fig. 11).

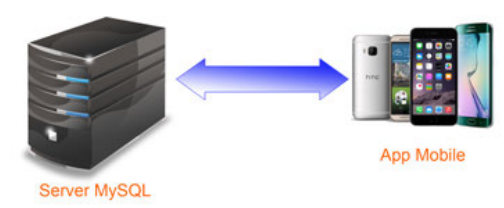

Figure 11. Data are not stored locally but are sent through the appropriate script for connection to a central server in a MySQL database 
Interactions with the designed application, for example virtual browsing, data addition, and connection with the database, are obtained through specific scripts written in C\# (Fig. 12) that manage the variables necessary to implement each action. For example, the script for graphic notes was created using a sequence of specific actions:

- $\quad$ Acquiring the screenshot of the user's viewpoint;

- $\quad$ Creating a plane perpendicular to the visual axis, with the same dimension as the screen of the mobile support;

- $\quad$ Applying the screenshot as plane texture;

- Creating the graphic note defined by the user with the touchscreen of the mobile support, using the raycast function. In particular, with this function, it is possible to send invisible rays, parallel to visual axis, in correspondence to the point touched by the user on the touchscreen. The intersection of these rays with the plane, created previously, identifies a series of spatial points to which a red circle is then associated. Because of the proximity of these circles with respect to their dimension, a continuous line can be simulated, which then leads to the creation of the graphic note;

- $\quad$ Acquiring the mobile-support screenshot and its saving in the SQL database.

Other scripts use the raycast function to identify the spatial coordinates of the points (essential to associate the information in the virtual space or to measure distances) or to identify the name of a GameObject (GameObjects are the fundamental objects in Unity that represent characters, props and scenery), which is useful to retrieve specific information in the database (Fig. 13).

Three-dimensional browsing requires the use of two pointers located the corners of the screen, allowing both the movement (left sticker) and the rotation (right sticker) of the user's viewpoint. Lastly, the three-dimensional models used in the present study derive from the three-dimensional surveying procedures, which were simplified with retopology and advanced texturing techniques. To avoid increasing the dimension of the installation starter pack, the three-dimensional models are downloaded the moment the user chooses the sample to be visualised, by then saving it in a specific folder of the mobile support, making it available to be subsequently visualised.

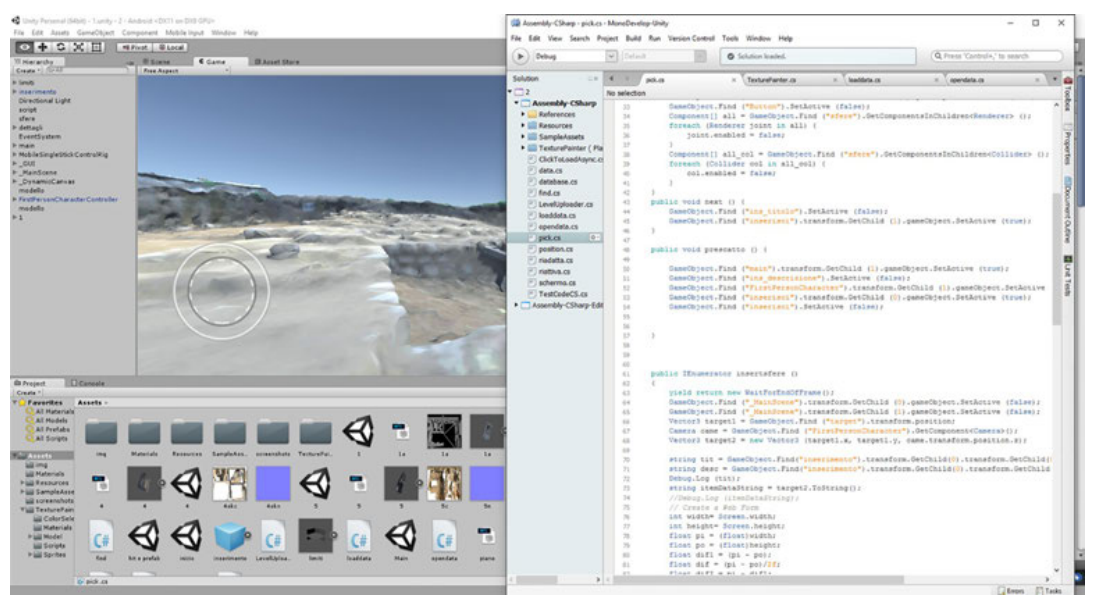

Figure 12. Use of Unity 3D to develop the mobile app. On the right: the programming window of the scripts in the C\# Language 



Figure 13. Some screenshots of the application: a) list of the excavations on the server side; $b$ ) pop-up menu with programmed features; c) VR view of the excavation; d) drawing on the snapshot; e) window to enter a title and description; f) snapshot stored server side; g) visualization of information entered; $h$ ) window with storage of a photo and title. 


\section{CONCLUSION}

The technology of representation is constantly developing, offering the user increasingly higher interactivity. Refined virtual-reality techniques and increasingly powerful technological tools are the fundamental requirements to create useful applications in the field of cultural heritage, which can help researchers to share and spread information. Technological tools and applications should not be regarded as distant from the discipline of cultural heritage. On the contrary, they facilitate researching and sharing operations and are much more efficient than traditional means.

Technologies for representation are today in continuous development and allow an ever-greater interactivity by the end user. Techniques of virtual reality increasingly refined and technological tools ever more powerful are the essential ingredients for making useful applications in the field of cultural heritage. The possibility of using these state-of-the-art tools can be of great interest to help researchers to share and disseminate information. These tools and technological applications should be seen as tools to facilitate the operations of research and sharing in a much more effective way than can be done with traditional methods.

\section{ACKNOWLEDGEMENTS}

The authors wish to thank: Valeria d'Aquino, Giacomo Baldini, GIANO snc, Paolo Nannini, Giovanni Roncaglia, Stefano Sarri, Pasquino Pallecchi, Domenico Zaccaria, the Municipality of Volterra, Stokholm University, Cassa di Risparmio di Volterra, Fondazione Cassa di Risparmio di Volterra and Franca Taddei.

The author would also acknowledge that paragraph 2 was written by E. Sorge, while C. Battini wrote the remaining paragraphs.

\section{REFERENCES}

Jean Angelo Beraldin. 2004. Integration of Laser Scanning and Close-Range Photogrammetry - The Last Decade and Beyond. In XXth International Society for Photogrammetry and Remote Sensing. Istanbul, Turkey, 972-983.

Stefano Bertocci. 2014. Un progetto per il rilievo digitale del sito archeologico di Masada, patrimonio UNESCO, in Israele. In Paolo Giandebiaggi \& Chiara Vernizzi, eds. Atti del $36^{\circ}$ convegno internazionale dei Docenti della Rappresentazione. Parma: Gangemi Editore, 549-556.

Alessandro Bezzi, Luca Bezzi, and Benjamin Ducke. 2011. Computer Vision e Structure from Motion, nuove metodologie per la documentazione archeologica tridimensionale: un approccio aperto. In Giuliano De Felice \& Maria Giuseppina Sibilano, eds. ARCHEOFOSS open source, free software e open format. Edipuglia, 103-111.

Wolfgang Boehler and Andreas Marbs. 2004. 3D Scanning and photogrammetry for heritage recording: a comparison. In Proceedings of the 12th International Conference on Geoinformatics. University of Galve, Sweden, 291-298.

Markus Grabner, Ralph Wozelka, Manish Mirchandani, and Konrad Schindler. 2003. Web-based visualization of virtual archaeological sites. In F.Niccolucci D. Arnold, A. Chalmers, ed. VAST 
2003: The 4th International Symposium on Virtual Reality, Archaeology and Intelligent Cultural Heritage. Brighton, United Kingdom, 1-12.

Pierre Grussenmeyer, Tania Landes, Thomas Voegtle, and Konrad Ringle. 2008. Comparison methods of terrestrial laser scanning, photogrammetry and tacheometry data for recording of cultural heritage buildings. In The International Archives of the Photogrammetry, Remote Sensing and Spatial Information Sciences. Beijing, 299-304.

Kevin Kelly, Adam Heilbrun, and Barbara Stacks. 1989. Virtual Reality: An Interview with Jaron Lanier. Whole Earth Rev. 64 (1989), 108-120.

Sofia Pescarin, Augusto Palombini, Luigi Calori, and Andrea Negri. 2009. Ambienti collaborativi 3D. Il caso di Virtual Rome. Archeol. e Calc. Suppl. 2 (2009), 121-130.

Marco Potenziani, Marco Callieri, Matteo Dellepiane, Massimiliano Corsini, Federico Ponchio, and Roberto Scopigno. 2015. 3DHOP: 3D Heritage Online Presenter. Comput. Graph. 52 (2015), 129-141.

Michele Russo, Fabio Remondino, and Gabriele Guidi. 2011. Principali tecniche e strumenti per il rilievo tridimensionale in ambito archeologico. Archeol. e Calc. 22 (2011), 169-198.

Vassilios Vlahakis et al. 2002. Archeoguide: An augmented reality guide for archaeology sites. IEEE Comput. Graph. Appl. 22(5) (2002), 52-59.

Received October 2016; revised September 2017; accepted November 2017. 